\title{
Assessment of bio-based materials for enhanced signal detection of sporozoites using csELISA
}

\section{Diego Gomez-Maldonado}

Auburn University

\section{Haley Stephens}

Auburn University

Alice C. Sutcliffe

CDC: Centers for Disease Control and Prevention

Maria Andrea Camarano Eula

Auburn University

Iris Beatriz Vega Erramuspe

Auburn University

Ellen M. Dotson

CDC: Centers for Disease Control and Prevention

Maria Soledad Peresin

Auburn University

Sarah Zohdy ( $\nabla$ sarahzohdy@gmail.com )

Auburn University

\section{Research Article}

Keywords: Malaria, Plasmodium, chitosan, cellulose nanocrystals, QCM-D, circumsporozoite, paperbased assays

Posted Date: January 28th, 2022

DOI: https://doi.org/10.21203/rs.3.rs-1082751/v1

License: (c) (i) This work is licensed under a Creative Commons Attribution 4.0 International License.

Read Full License 


\section{Assessment of bio-based materials for enhanced signal detection of sporozoites using csELISA}

Diego Gomez-Maldonado ${ }^{1,2^{*}}$, Haley Stephens ${ }^{1 *}$, Alice C. Sutcliffe ${ }^{3}$, Maria Andrea Camarano Eula ${ }^{1}$, Iris Beatriz Vega Erramuspe ${ }^{1,2}$, Ellen M. Dotson ${ }^{3}$, Maria Soledad Peresin ${ }^{1,2}$, Sarah Zohdy $^{1,4 \dagger}$.

${ }^{1}$ School of Forestry and Wildlife Sciences, Auburn University, Auburn, AL, United States, ${ }^{2}$ Forest

Products Development Center, School of Forestry and Wildlife Sciences, Auburn University, Auburn, AL,

United States, ${ }^{3}$ Centers for Disease Control and Prevention, Atlanta, GA, United States, ${ }^{4}$ College of

Veterinary Medicine, Auburn University, Auburn, AL, United States

*These authors contributed equally to this work

$\dagger$ Corresponding author, current address: President's Malaria Initiative (PMI), US Centers for Disease Control and Prevention, Atlanta, GA, USA 


\begin{abstract}
Background:

Malaria is responsible for over 435,000 deaths annually, with most cases occurring in sub-Saharan Africa. Detecting the presence of Plasmodium spp. sporozoites (spzs) in the salivary glands of Anopheles vectors of the parasites using the circumsporozoite enzyme-linked immunosorbent assay (csELISA) is an important malaria surveillance method. The addition of bio-based materials have shown the potential to improve the adsorption and binding of target antigens and thus can improve the sensitivity and detection of analytes in immunoassays. Here, we evaluate the use of two bio-based polymers, chitosan, and cellulose nanocrystals (CNC), as antibody carriers and substrate coating on 96-well plates and on a paper substrate to determine whether detection of Plasmodium falciparum (Pf), P. vivax VK210 (Pv210) and P. vivax VK247 (Pv247) can be improved through assay modification.

Methods:

Modified csELISA assays were conducted using chitosan and CNC as either an antibody carrier or a well coating on 96-well plates (ultra-low- and high-affinity) and results read using standard spectrophotometry of 96-well plates and a quantitative image-based color analysis using photographs of paper plate assays. Changes in frequency and dissipation, resulting from the adsorption of antibodies to model films in a quartz crystal microbalance with dissipation monitoring (QCM-D), were followed to understand better the interactions between the bio-based materials and assay proteins.

\section{Results:}

The csELISA performed on high-binding well-plates showed that chitosan, used either as an antibody carrier or well coating, resulted in the greatest increase in detection for Pv210 and Pv247 positive recombinant proteins, increasing absorbance readout values up to $6 \mathrm{x}$ for Pv210 and up to 5x for Pv247. On paper csELISA (PcsELISA), chitosan as an antibody carrier yielded the greatest increase in detection sensitivity for all three Plasmodium species when color intensity of positive recombinant proteins was compared to blanks. Compared to controls, chitosan as a carrier resulted in a $\sim 2.5$-fold increase in color intensity for Pf, a 4-fold increase for Pv210, and a 2-fold increase for Pv247. QCM-D showed a preferred interaction between the assay antibodies and chitosan surfaces, most likely driven by electrostatic interactions.

\section{Conclusion:}

The addition of bio-based materials, mainly chitosan, as shown by QCM-D interactions, absorbance readout values, and image-based color analysis, increased the color intensity of positive samples run through csELISA in all systems, allowing for clearer detection of Plasmodium spp visually, using a spectrophotometer and quantitative color intensity. Furthermore, the adaptation of a PcsELISA coated with chitosan using positive recombinant proteins shows potential as a cost-effective alternative assay platform as it reduced reagent volumes by $80 \%$ and assay run time from seven hours to one hour.
\end{abstract}

Keywords: Malaria, Plasmodium, chitosan, cellulose nanocrystals, QCM-D, circumsporozoite, paperbased assays. 


\section{Background}

44 Human malaria is a life-threatening disease caused by Plasmodium, a group of apicomplexan parasite 45 species that is vectored by female Anopheles mosquitoes (1). Detecting the presence of Plasmodium spp. 46 sporozoites in the salivary glands of Anopheles is an important malaria surveillance method, which helps 47 measure the intensity of malaria transmission and evaluate intervention methods to assess programmatic impact (2).

The current gold standard for sporozoite detection in infected mosquitoes is the enzyme-linked immunosorbent assay (ELISA) (3). Specifically, the circumsporozoite (cs) sandwich ELISA utilizes a capture and detection antibody complex to identify the circumsporozoite protein, the major surface protein of the sporozoite (4), and has been used globally as an entomological indicator for the detection of infectious mosquito vectors.

The csELISA provides high sensitivity and specificity for Plasmodium detection (5). Despite these advantages and the relatively low cost associated with csELISAs when compared to molecular techniques, there are some disadvantages. Assays are run in 96-well plates or tubes, requiring large volumes of sample and reagents for optimal performance. Due to cross-reactivity, non-specific binding of proteins to the capture antibodies may also result in false positives (6), requiring an additional boiling step for confirmation. The csELISA technique is time-consuming, requiring more than seven hours to complete, with multiple incubation steps of 30 minutes to two hours. Additionally, a microplate reader is traditionally required to measure the colorimetric readout (7), and these devices can cost up to \$20,000 USD without taking into consideration maintenance and potential future software upgrades.

64 been developed as a base immunoassay, including adaptations of ELISAs. Paper-based ELISAs offer a suitable alternative in terms of cost reduction, ease of use, and conservation of assay reagents and samples (8). Paper-based assays eliminate the need for a plate reader, and alternatives such as cellphone imaging 
are deposited directly onto the paper substrate rather than into the well of a 96-well plate, the surface area of the reaction is reduced, thereby decreasing the volume of antibodies, samples and other reagents used (9). Despite the numerous advantages that come with paper-based assays, there are limitations, notably in those that use a peroxidase-based reaction as colorimetric indicator. Due to differences in capillary forces among the reagents and analytes, uneven distribution over the test zone can result in heterogenous color formation, making correct interpretation of the results difficult (10).

To overcome this lack of homogeneity and improve binding affinity between the antibodies and the test zones, as well as to act as coating agent smoothing the surfaces (and thus capillary forces), biobased materials can be used as additives in the process. Some of these bio-based materials have been demonstrated to improve biomolecule stability and immobilization on the well walls (11). The goal of this work was to assess if the addition of these materials can improve assay sensitivity and reduce detection limits when compared to traditional 96-well plate based ELISAs.

One such bio-based material is cellulose. This polymer is formed by glucose monomer links in $\beta$ $(1 \rightarrow 4)$-D-glucopyranose conformation, and it is the most abundant polysaccharide on earth, present in the cell walls of plants and wood, found in tunicates and algae, and is also produced by some bacteria (12) Fibrils are packed in two alternating forms within the cellulose macrostructure: a tight crystalline form, where cellulose chains are highly ordered, and an amorphous one. Traditional processing of wood results in lignocellulosic pulp, which is the raw material to produce paper (13). However, after an acid hydrolysis step, the pre-treated lignocellulosic pulp can be processed to selectively remove the amorphous portions and produce cellulose nanocrystals (CNC) (14).

Like cellulose, chitosan has also shown potential as a bio-based material for assay improvement. Chitosan is a polycationic linear polysaccharide which is easily obtained from chitin, the second most abundant natural polysaccharide after cellulose. Chitosan is produced by the partial deacetylation of chitin under alkaline conditions, which is a structural component of the exoskeleton of arthropods and cell walls

92 of fungi and yeast (15-17). This biodegradable, nontoxic and biocompatible bio-based material has 
93 demonstrated a key role in paper-based assays, producing uniform color signals at the detection spot when 94 used as a substrate $(18,19)$.

Here, the chemistry of bio-based materials was integrated with the well-established csELISA to determine whether this could potentially improve the detection of sporozoites of $P$. falciparum, $P$. vivax

97210 and P. vivax 247. To better understand the interactions of the bio-based materials with the antibodies

98 used in the assays, Quartz Crystal Microbalance with Dissipation monitoring (QCM-D) was used to analyze

99 the adsorption of the assay antibodies to thin films of the bio-based materials, where frequency and 100 dissipation shifts were used to quantify the adsorption capability as an indicator of the strength of the 101 interactions.

\section{Methods}

\section{Materials and reagents}

104 Two bio-based materials were selected based on their binding properties to antibody proteins: chitosan 105 (85\% deacetylated, Alfa Aesar, Cat. $\mathrm{N}^{\circ}$ : J64143) and cellulose nanocrystals (CNC, 10.7\% wt., 2016-FPL106 CNC-098, Process Development Center, The University of Maine). All capture and detection antibodies, 107 positive controls (recombinant proteins), and substrates were obtained from BEI Resources (Cat no. MRA108 890; MRA-1028K)(20). These recombinant proteins have been used in other studies adapting the csELISA 109 to other assay platforms, such as multiplex bead-based immunoassays (21). The P. falciparum positive 110 control antigen is derived from recombinant protein R32tet 32 produced in Escherichia coli (Smith Kline 111 and French Laboratories, USA) (22). The P. vivax210 and P. vivax247 positive control antigen, PvCSPv1, 112 is a recombinant fusion protein that includes cs regions of both $P$. vivax 210 and $P$. vivax 247 produced in 113 Pichia pastoris (Protein Potential, USA) (23). All antibodies and positive controls were received 114 lyophilized and were rehydrated to the concentrations indicated in the kit instructions. These recombinant 115 proteins were used as positive controls and will be referred to as "recombinant proteins" from this point 116 onward. 
Capture antibodies were anti-sporozoite monoclonal antibodies (mAb) against three target

118 antigens: regions of the Plasmodium falciparum (Pf), P. vivax-210 (Pv210), and P. vivax 247 (Pv247)

119 circumsporozoite (cs) proteins. Detection antibodies were obtained already conjugated to horseradish

120 peroxidase (HRP). Stock concentrations of Pf, Pv210, and Pv247 recombinant proteins were $100 \mathrm{pg} / \mu \mathrm{L}$,

$1219100 \mathrm{pg} / \mu \mathrm{L}$, and $4550 \mathrm{pg} / \mu \mathrm{L}$, respectively. Working recombinant protein solutions were made by diluting

122 the stock solutions in blocking buffer to $2 \mathrm{pg} / \mu \mathrm{L}, 182 \mathrm{pg} / \mu \mathrm{L}$, and $91 \mathrm{pg} / \mu \mathrm{L}$ for Pf, Pv210 and Pv247,

123 respectively. The blocking buffer was a solution of $0.5 \%$ casein in phosphate-buffered saline (PBS, $\mathrm{pH} 7.4)$,

124 and the wash buffer was a solution of $0.05 \%$ Tween 20 in PBS, pH 7.4. Bio-based materials were used in

125 assays as either well-coating or antibody-carrier or in combination on the same plate. The csELISA protocol

126 used was obtained from BEI Resources (20).

127 Testing of bio-based materials on high- and ultra-low binding 96-well plates

Circumsporozoite ELISA was performed on four types of clear polystyrene 96-well microplates.

129 The four plates were divided into two categories: high binding plates (Corning 3366, round bottom, and

130 MaxiSorp ${ }^{\mathrm{TM}} / \mathrm{Nunc}-$ Immuno, flat bottom), and ultra-low binding plates (Corning® 7007, round bottom and

131 Corning® 3474, flat bottom). Absorbance readings of all plates were performed using a SpectraMax 190

132 spectrophotometer system from Molecular Devices (Silicon Valley, CA, U.S.). Both chitosan and CNC

133 were tested as well-coating materials for the plates and as antibody carriers. When used as antibody carriers,

134 the polymers were mixed with the antibodies in solution before adding to the plates.

\section{Preparation of bio-based materials for well coating}

For well-coating solutions, a $0.1 \%$ chitosan solution and a $0.1 \% \mathrm{CNC}$ suspension were prepared in $1 \%$ acetic acid and water, respectively. All suspensions and solutions were prepared with Milli-Q water

138 (Millipore Inc., $18.2 \mathrm{M} \Omega \mathrm{cm}$ ). Suspensions of $\mathrm{CNC}$ were sonicated for 5 minutes at $25 \%$ amplitude, with

139 the pulser set in five seconds intervals of three second "on" and two seconds "off," while maintaining the

140 suspension in an ice bath, prior to use. Wells of the 96-well polystyrene plates were filled with $200 \mu \mathrm{L}$ of

141 the bio-based material, covered with a lid and incubated for 30 minutes at room temperature. Thereafter,

142 the solution, or suspension, was aspirated and the wells were rinsed as follows: i) wells coated with chitosan 
$0.1 \%$ were rinsed one time with $200 \mu \mathrm{L}$ of $1 \%$ acetic acid and two times with $200 \mu \mathrm{l}$ MilliQ-water; ii) wells

144 coated with $0.1 \%$ CNC were rinsed one time with $200 \mu \mathrm{L}$ of PBS-Tween $(0.05 \%)$ and two times with

$145200 \mu \mathrm{L}$ MilliQ-water. After that, the plates were left covered with plate lids overnight at room temperature

146 (ca. $20^{\circ} \mathrm{C}$ ) to air dry.

$147 \quad$ Preparation of bio-based materials for antibody carriers

148 A $1 \%$ chitosan solution was prepared in $1 \%$ acetic acid and then diluted to $0.1 \%$ with PBS, pH 7.4.

149 CNC suspensions were diluted in PBS, pH 7.4 to obtain a $0.1 \%$ concentration and sonicated, as previously

150 described. The Pf , Pv210 or Pv247 stock capture antibodies were rehydrated as described (20), and added

151 to $5 \mathrm{~mL}$ of the prepared bio-based material solution (in the place of PBS). Antibody-bio-based material

152 solutions were then vortexed and incubated at room temperature for one hour before use. The remainder of

153 the protocol was followed as described in the MR4 csELISA protocol (20).

\section{Quartz Crystal Microbalance with Dissipation monitoring (QCM-D)}

155 To better understand the interactions occurring between the bio-based materials and the csELISA 156 antibodies, adsorption of the Pf, Pv210 and Pv247 antibodies on model films was performed in a QSense 157 Analyzer from Biolin Scientific (Västra Frölunda, Västra Frölunda, Sweden). Model films were prepared 158 over polyethyleneimine (PEI) precoated gold quartz sensors ( $5 \mathrm{MHz}$ base resonance) by spin coating 180 $159 \mu \mathrm{L}$ of $0.1 \%$ solutions of the bio-based materials at $3000 \mathrm{rpm}$ for $1 \mathrm{~min}$ in a WS-650MZ-23NPPB spin coater 160 by Laurell Technologies Corporation (North Wales, PA, U.S.). For measurements, the sensors were first 161 stabilized on PBS buffer (pH 7.4) at $25{ }^{\circ} \mathrm{C}$ with a flow of $50 \mu \mathrm{L} / \mathrm{min}$. Then, $4 \mu \mathrm{L} / \mathrm{mL}$ of Pf or $8 \mu \mathrm{L} / \mathrm{mL}$ of 162 Pv210/Pv247 antibody solutions were poured into the channel and allowed to interact with the surfaces 163 until no changes were detected. Finally, a rinse with PBS was performed to remove the excess material. 164 Data analysis was performed using with DFind Software from Biolin Scientific (Västra Frölunda, Sweden) 165 using the Composite Sauerbrey model and the software's predetermined density values.

\section{Development of paper ELISA template}

167 A paper-mimic of 96-well plates (Figure 1) was created on chromatography paper (Whatman Grade

1681 Chr cellulose chromatography paper, from Cytiva, at Marlborough, MA, U.S.). Circular zones $6.5 \mathrm{~mm}$ in 
170 a commercial liquid wax solution, wells were then outlined using a fine-tip paintbrush. To create a 171 hydrophobic well-barrier, the "plate" was heated on a hot plate at $100^{\circ} \mathrm{C}$ for three minutes to allow for 172 complete penetration of the wax through the paper matrix. Chitosan and CNC were then evaluated on the 173 paper 'plates' in different combinations as antibody carriers and well-coating. Recombinant proteins and 174 bio-based materials were prepared as described above. For well-coatings, $100 \mu \mathrm{L}$ of bio-based material 175 solution or suspension was pipetted onto paper wells and allowed to dry overnight. For bio-based material 176 antibody carrier solutions, $10 \mu \mathrm{L}$ was pipetted onto each well.

\section{Paper cs-ELISA protocol adaptation}

All incubation steps from the csELISA protocol (20) were reduced to 15 minutes. Excess reagent was removed after 15 minutes by placing a Kimwipe (Kimtech Science Kimwipes Delicate Task Wipes product code \#34155) on the underside of the paper plate, which wicked liquid through. To prevent leaking placing it on an empty reagent reservoir. All volumes were reduced to $20 \%$ of the volume outlined in the the entire paper-plate, to create an image to assess the resulting color change. concentration, a two-fold dilution of the stock concentration, the reported working solution concentration,

190 and a ten-fold dilution of the working concentration. Pf: $100 \mathrm{pg} / \mu \mathrm{L}$ (stock), $50 \mathrm{pg} / \mu \mathrm{L}, 2 \mathrm{pg} / \mu \mathrm{L}$ (working),

191 and $0.2 \mathrm{pg} / \mu \mathrm{L} . \mathrm{Pv} 210: 9.1 \mathrm{ng} / \mu \mathrm{L}, 4.55 \mathrm{ng} / \mu \mathrm{L}, 182 \mathrm{pg} / \mu \mathrm{L}$, and $18.2 \mathrm{pg} / \mu \mathrm{L} . \mathrm{Pv} 247: 4.55 \mathrm{ng} / \mu \mathrm{L}, 2.275$

$192 \mathrm{ng} / \mu \mathrm{L}, 91 \mathrm{pg} / \mu \mathrm{L}$, and $9.1 \mathrm{pg} / \mu \mathrm{L}$.

\section{CYMK Analysis for Paper}


A calibration curve was prepared to convert the observed color change into numerical data. Briefly,

195 photos taken 15 minutes after adding ABTS were uploaded into photo editing software (Adobe Photoshop

196 CC 2019) and analyzed using the Cyan-Yellow-Magenta-Black (CYMK) color processing. The average

197 color of each well was obtained by selecting the well-borders and applying a filter (Filter -> Blur ->

198 Average) that takes the average color of a selection and replaces the original selection with the color average

199 (Figure 1). The CYMK color values were then recorded for each selection and plotted to compare color

200 intensity changes associated with the pure recombinant protein concentrations (Figure 1). As the

201 concentration of the recombinant proteins increased, greater differences in the percent color intensity were

202 seen, which were associated with a more visible difference in color between concentrations.

204 Results

\section{Bio-based materials on high- and ultra-low-binding 96-well plates}

$206 \boldsymbol{P f}$

For assays assessing Pf antibodies, none of the $\mathrm{CNC}$ or chitosan combinations as antibody carriers

or plate coatings on high-affinity binding and ultra-low binding plates displayed a significant improvement

209 in the detection of Pf recombinant proteins at the working concentration $(2 \mu \mathrm{g} / \mu \mathrm{L})$. For high-binding plates

210 (Corning 3366 and MaxiSorp ${ }^{\mathrm{TM}}$ ), the control wells with the recombinant proteins exhibited the greatest

211 absorbance values compared to the wells with samples where the bio-based materials were used. On ultra-

212 low binding plates (Corning® 7007 and Corning® 3474), the absorbance values for all samples, including

213 control wells without bio-based material used, were very low (Figure 2).

$214 P v 210$

215 In the case of Pv210, the use of chitosan enhanced the detection sensitivity of the recombinant 216 proteins on high-binding and ultra-low binding plates. When Pv210 recombinant proteins were tested at the 217 working concentration $(182 \mu \mathrm{g} / \mu \mathrm{L})$, assays performed with chitosan as well-coating or antibody-carrier 218 showed absorbance values up to six times higher than those from the control wells (without chitosan as a 
well-coating or antibody carrier) (Figure S1). Moreover, on high-affinity binding plates, the absorbance

220 values of Pv210 from wells where chitosan was used as a well-coating, antibody carrier or when used at

221 the same time with $\mathrm{CNC}$ as a carrier at recombinant protein concentration of $18.2 \mu \mathrm{g} / \mu \mathrm{L}$ were comparable

222 to those with Pv210 at $182 \mu \mathrm{g} / \mu \mathrm{L}$ in wells where bio-based materials were not used as a well-coating or

223 antibody carrier. Thus, using chitosan, as well-coating or antibody increased the sensitivity of recombinant

224 protein concentration detection by 10-fold (Figure Error! Reference source not found.2).

225 Circumsporozoite ELISA control assays (no bio-based materials as antibody carriers or well-

226 coatings) performed on ultra-low binding plates for Pv210 yielded no increase in absorbance values when

227 recombinant protein was present over the dilution series, compared to blanks (blocking buffer only) (Figure

228 2), indicating that little to no antibody binding occurred on the well surfaces. Nevertheless, the absorbance

229 values obtained when chitosan was used either as an antibody carrier or well-coating were between 1.2 and

$2301.7 x$ higher than those observed for Pv210 on control wells of high-affinity binding plates.

For Pv210 assays performed with CNC as a well-coating, results more heavily depended on the

232 specific plate type, and did not yield an increased absorbance value over the recombinant protein or when

233 the chitosan was used alone either as an antibody carrier or well-coating. With CNC applied as a well-

234 coating with chitosan as an antigen carrier on the high-binding plate a two-fold increase in absorbance was

235 observed when compared to controls.

$236 \quad P v 247$

237 The results for Pv247 on high-affinity binding plates showed a similar trend to that observed for Pv210

238 (see Figure 2), with up to a five-fold increase in absorbance values when compared to controls when

239 chitosan was used as an antibody carrier or well-coating. Chitosan, either as a well-coating or antibody

240 carrier, was also capable of reducing the working concentration, and lowering the detection limit, of the

241 Pv247 recombinant protein up to five-fold without a significant reduction in sensitivity for assays carried

242 out on both high-binding plates (Figure 2). 
245 QCM-D followed the adsorption of the capture antibodies onto model films of the bio-based materials to 246 better understand the affinity between them. Flow concentrations of the antibodies were $2 \mu \mathrm{g} / \mu \mathrm{l}$ for Pf, 182 $247 \mu \mathrm{g} / \mu \mathrm{l}$ for Pv210, and $91 \mu \mathrm{g} / \mu \mathrm{l}$ for Pv247 in PBS and flow of $50 \mu \mathrm{L} / \mathrm{min}$. The graphs of results are shown

248 in Figure 3, and a summary of the relevant values is presented in Table 1. Table 1 also presents the shifts 249 after washing with PBS, as these values are the irreversible mass attached to the surface of the thin films.

250 With Pf, Pv210 and Pv247antibodies, chitosan presented higher frequency drops than CNC, indicating a

251 better interaction between the antibodies and chitosan over that of CNC. The Pf antibody appeared to 252 interact more strongly with both materials contrary to the observed performance in 96-well plates. The Pf 253 antibody was the only antibody of the three with multiple layers adsorbed, as demonstrated by the multiple 254 changes of slope in the frequency and dissipation plots (Figure 3B). These multilayers may reduce the 255 available sites for antibody binding with the antigen. For Pf, chitosan had 2.3-fold higher adsorption of the antibodies when compared to CNC adsorption, while for Pv210 and Pv247 the frequency and mass shifts were similar for both chitosan and CNC.

Table 1: Data extracted from QCM-D of irreversible adsorbed antibodies on model films of cellulose nanocrystals (CNC) and chitosan surfaces.

\begin{tabular}{|c|c|c|c|c|c|c|c|c|c|}
\hline & \multicolumn{3}{|c|}{ Pf } & \multicolumn{3}{|c|}{ Pv210 } & \multicolumn{3}{|c|}{ Pv247 } \\
\hline $\begin{array}{l}\text { Model } \\
\text { film }\end{array}$ & $\begin{array}{c}\Delta \mathrm{F} \\
{[\mathrm{Hz}]}\end{array}$ & $\begin{array}{c}\Delta \mathrm{D} \\
{[\mathrm{ppm}]}\end{array}$ & $\begin{array}{c}\Delta \mathrm{m}_{\text {Sauerbrey }} \\
{\left[\mathrm{ng} / \mathrm{cm}^{2}\right]}\end{array}$ & $\Delta \mathrm{F}[\mathrm{Hz}]$ & $\Delta \mathrm{D}[\mathrm{ppm}]$ & $\begin{array}{c}\Delta \mathrm{m}_{\text {Sauerbrey }} \\
{\left[\mathrm{ng} / \mathrm{cm}^{2}\right]}\end{array}$ & $\begin{array}{c}\Delta \mathrm{F} \\
{[\mathrm{Hz}]}\end{array}$ & $\begin{array}{c}\Delta \mathrm{D} \\
{[\mathrm{ppm}]}\end{array}$ & $\begin{array}{c}\Delta \mathrm{m}_{\text {Sauerbrey }} \\
{\left[\mathrm{ng} / \mathrm{cm}^{2}\right]}\end{array}$ \\
\hline $\mathrm{CNC}$ & $\begin{array}{l}-10.25 \\
\pm 0.03\end{array}$ & $\begin{array}{c}0.51 \pm \\
0.01\end{array}$ & $\begin{array}{c}180.3 \pm \\
0.2\end{array}$ & $\begin{array}{c}-6.28 \pm \\
0.03\end{array}$ & $\begin{array}{c}0.22 \pm \\
0.01\end{array}$ & $\begin{array}{l}117.0 \\
\pm 0.2\end{array}$ & $\begin{array}{c}-4.20 \pm \\
0.02\end{array}$ & $\begin{array}{c}0.11 \pm \\
0.01\end{array}$ & $79.1 \pm 0.2$ \\
\hline Chitosan & $\begin{array}{l}-23.93 \\
\pm 0.02\end{array}$ & $\begin{array}{c}1.77 \pm \\
0.01\end{array}$ & $\begin{array}{c}394.8 \pm \\
0.1\end{array}$ & $\begin{array}{c}-7.30 \pm \\
0.02\end{array}$ & $\begin{array}{c}0.13 \pm \\
0.01\end{array}$ & $\begin{array}{l}136.0 \\
\pm 0.2\end{array}$ & $\begin{array}{c}-4.34 \pm \\
0.02\end{array}$ & $\begin{array}{l}0.35 \\
0.01\end{array}$ & $77.2 \pm 0.2$ \\
\hline
\end{tabular}




\section{Bio-based materials as antibody carriers and well-coatings with the Paper csELISA}

CYMK data was compared between seven different assays for three antibodies: chitosan wellcoating and $\mathrm{CNC}$ antibody carrier combination, $\mathrm{CNC}$ well-coating and chitosan antibody carrier

264 combination, chitosan well-coating, CNC well-coating, chitosan antibody carrier, CNC antibody carrier, 265 and a control (no antibody nor carrier and no bio-based well-coating).

$266 P f$

For Pf recombinant proteins tested at the working concentration $(2 \mu \mathrm{g} / \mu \mathrm{L})$, the combination of

268 chitosan as an antibody carrier and $\mathrm{CNC}$ as a well-coating yielded an approximately 4.5 -fold increase in

269 CMYK color intensity (with blank/background values subtracted) compared to controls (no antibody carrier 270 or well-coating). Similar increases were observed from the other two CNC assays, with CNC as an antibody 271 carrier or well-coating alone. Chitosan as an antibody carrier alone provided an approximately 2-fold 272 increase in color intensity when compared to controls (no antibody-carrier or well-coating; Figure 4 and 273 S2).

When ten-fold dilutions were performed, $\mathrm{CNC}$ as a well-coating and chitosan as an antibody carrier continued to perform best at lower concentrations, followed closely by $\mathrm{CNC}$ as either a well-coating or 276 antibody carrier (Figure 4).

$277 \quad P v 210$

At the recombinant protein working concentration, the top three performing bio-based polymer 279 assays involved an antibody carrier (Figure 4). Two of them had chitosan as the antibody carrier, yielding 280 a 4-4.5-fold increase in color intensity when compared to controls (no antibody carrier or well-coating). 281 Chitosan as an antibody carrier alone increased color intensity approximately 4-fold, while chitosan as an 282 antibody carrier in combination with $\mathrm{CNC}$ as a well-coating and $\mathrm{CNC}$ as an antibody carrier alone provided 283 an approximate 4.5-fold increase in color intensity.

At the point following the working concentration (first dilution of antigens Pv210 tested), similar 285 increases for chitosan as an antibody carrier in combination with CNC as a well-coating as well as CNC as 
a well-coating alone were observed, with $\mathrm{CNC}$ as a well-coating providing the greatest increase in intensity.

287 Chitosan as an antibody carrier alone performed better than controls (no antibody carrier or well-coating)

288 but did not perform as well as the first two which involved CNC.

$289 \quad P v 247$

For the Pv247 recombinant protein assayed at the working concentration, the same three assays that 291 performed optimally with Pv210 antibodies and recombinant proteins performed the best. (Figure S2).

292 Chitosan as an antibody carrier alone yielded the greatest increase in color intensity, yielding an 293 approximate 2-fold increase in color intensity when compared to controls (no antibody carrier or well294 coating; Figure 4). Chitosan as an antibody carrier in combination with CNC as well-coating and as CNC 295 as a well-coating alone performed similarly, yielding slightly less than a 2-fold increase of color intensity when compared to controls (no antibody carrier or well-coating).

When ten-fold dilutions were performed, a similar trend was observed as for the other antigens: $\mathrm{CNC}$ as a well-coating performed the best at lower concentrations, with chitosan as an antibody carrier and the combination of chitosan as an antibody carrier with $\mathrm{CNC}$ as a well-coating performing just below.

\section{Discussion}

In this study, two bio-based polymers, chitosan and CNC, were used to modify the csELISA and any resulting differences in detection capabilities were assessed. The use of low quantities $(<0.1 \%$ wt. of the solutions used) of both bio-based materials was shown to improve detection signals. Chitosan as an antibody carrier performed the best overall in improving detection on polystyrene plates regardless of highor ultra-low binding affinity. The favored interaction of chitosan with the antibodies was also observed on the QCM-D, where the surface it generated showed higher affinity for the antibodies. These results are 
electrostatic interactions and hydrogen bonding with different polymers, which may explain the increased

311 affinity observed when used with the commercially treated plates.

312 Thus, these results support the addition of chitosan as either a well-coating or antibody carrier in

313 the csELISA to help increase the intensity of the signal when detecting Pv210 and Pv247 proteins. This

314 may be particularly useful when determining the presence of sporozoites in field collected mosquitoes,

315 where the number of sporozoites can be highly variable (25).

316 Furthermore, the csELISA for malaria parasite detection in mosquitoes was adapted to a paper

317 format using the same bio-based materials as well-coatings and antibody carriers, thus combining the paper-

318 based csELISA, which reduced the volumes of reagents and samples necessary, with the improvements

319 observed using the bio-based materials.

Combining bio-based materials with paper-based assays provided advancements regarding the detection and visual readability of adapted csELISAs using cost-effective materials. The data presented here can be used to further develop and improve paper-based assays. Figure 5 shows photos taken of PcsELISAs after 15 minutes of drying and after drying overnight. Despite the higher intensity values seen

324 in assays with CNC as a well-coating, further optimization of bio-based materials may be needed to obtain consistently reproducible results. In assays where CNC was used as a well-coating (Fig 5. c-1), reagents tended to leak through the hydrophobic barriers, false positives were common, and overall color intensity

327 was greater. This made results harder to distinguish using the naked eye. Using CNC with a different paper 328 substrate, for example, nitrocellulose or nanocellulose coated papers, may provide a viable alternative to 329 prevent reagent leaking while maintaining color intensity. Color homogeneity, which can best be seen 330 following overnight drying (Fig. 5 x-2), was improved for both chitosan and CNC when compared to the 331 control (no antibody carrier or well-coating) (Fig. 5 a-1). The chitosan antibody carrier assay (Fig. 5 b-1) 332 showed a much clearer, even distribution of color throughout the entire test well. The change in color 333 intensity as recombinant protein concentration increased was easily visible to the naked eye for assays with 334 chitosan as antibody carrier. This is an important consideration, particularly if plate readers are not 335 available. Here, the chitosan antibody carrier paper-based csELISA was shown to have the most potential 
to be further explored for future use and scalability. This is due to consistent quantitative colorimetric data and clear visual results. Chitosan as an antibody carrier does not require the additional overnight incubation step that is necessary to coat paper 'plates'. Future work evaluating the use of paper-based csELISAs will reveal whether this method can be implemented for more economical and efficient detection of sporozoites

340 in field-collected mosquitoes in resource-limited settings.

The more favorable use of chitosan as an antibody carrier rather than a well-coating is likely related

342 to the more efficient interaction between the antibodies and chitosan when put in contact with the solution.

343 Specifically, more active points of chitosan can interact with the antibodies when mixed in solution instead

344 of those sites interacting with the paper or plate surfaces. This effect of groups exposed would also affect

345 the surface available to interact with the antibodies when being applied as the coating, as the surface area 346 of the bio-based materials will become more compact during the adsorption and drying (26). Furthermore,

347 the improved detection of the recombinant proteins when using the $\mathrm{CNC}$ as a well-coating and chitosan as 348 an antibody carrier can also been explained by the increase in surface area that the CNC coating induced 349 on the paper csELISA, which can be measured by the changes in rugosity and porosity of the paper (27).

350 This change in surface area could also increase the interaction between the chitosan and the cellulosic 351 surfaces - either the paper by itself, or the paper coated with CNC - in the presence of PBS, as the greater 352 salt content promotes adsorption between the two bio-based materials, chitosan and paper (cellulose)(28).

\section{Conclusions}

For Pv210 and Pv247, results of the experiments conducted on 96-well plates showed that chitosan

356 improved the detection of the recombinant proteins, as demonstrated by the higher absorbance values 357 observed when compared to controls (no antibody carrier or plate coating). This is potentially due to a 358 higher affinity between the chitosan and antibodies than the antibodies with the well surfaces. This improved interaction was also demonstrated by QCM-D, where adsorption of the antibodies was higher on the chitosan model films. Experiments with PcsELISAs showed the same trend, where the use of chitosan 
361 as either an antibody carrier or well-coating increased intensity values when compared to the controls (no

362 antibody carrier or well-coating). The use of CNC as a well-coating with PcsELISAs also improved the

363 signal further when compared with the test zones with no bio-based material, possibly by increasing the

364 available surface area for antibody-substrate interactions. Unlike the addition of chitosan which changed

365 the charge of the wells in polystyrene plates, CNC is a 3-D material which added volume and area, another

366 benefit to paper-based assays when compared to polystyrene plates.

367 The bio-based materials tested, particularly chitosan, both as antibody carriers and well-coatings,

368 have demonstrated their feasibility in improving the detection of the sporozoite proteins from $P$.

369 falciparum, P. vivax 210 and P. vivax 247 when used as an additional step in csELISA. Furthermore, this

370 work demonstrated the capability of chitosan and CNC to not only improve detection in traditional

371 polystyrene plates (both high- and ultra-low affinity binding), but also with paper-based csELISAs.

372 Although further development of PcsELISAs is needed, this could be a more convenient assay with

373 regard to cost, availability, scalability, time, and ease-of-use.

374 Abbreviations

375 CNC: Cellulose nanocrystals

376 csELISA: Circumsporozoite enzyme-linked immunosorbent assay

377 PcsELISA: Paper circumsporozoite enzyme-linked immunosorbent assay

378 spz: Sporozoites

379

380 Declarations

381 Ethics approval and consent to participate

382 Not applicable

383 Consent for publication

$384 \quad$ Not applicable

385 Availability of data and materials 
All data are made available in the manuscript and supporting documentation.

387 Competing interests

388 The authors declare no competing interests.

389 Disclaimer

390 The findings and conclusions in this manuscript are those of the authors and do not necessarily 391 represent the views of the CDC. Use of trade names is for identification only and does not imply 392 endorsement by the Centers for Disease Control and Prevention, the Public Health Service, or the 393 US Department of Health and Human Services.

\section{Funding}

395 This work was supported by a CDC innovation fund (iFund) award and the School of Forestry and 396 Wildlife Sciences and the Office of the Vice President for Research (OVPR) intramural grant 397 program (IGP) award at Auburn University. Laboratory support was provided by the US Centers 398 for Disease Control and Prevention Malaria Research and Reference Reagent and Resource Center 399 (MR4) and BEI Resources for providing the Plasmodium falciparum and P. vivax 210 and 247 400 Sporozoite ELISA Reagent Kits.

$401 \quad$ Author's contributions

402 AS, ED, SP, SZ conceived the study and participated in its design and coordination. HS, DGM, ACE 403 conducted the laboratory work. HS, DGM, ACE analyzed the data. HS, DGM, AS, and SZ wrote the 404 manuscript and created figures. DGM, HS, AS, ACE, IBVE, ED, SP, SZ participated in data analysis and 405 interpretation. All authors read and approved of the final manuscript.

406 Acknowledgements

407 The team is grateful to the Auburn University IGP program for providing support and collaborative 408 opportunities for this work. The authors also thank

409

410 Authors' information 
Diego Gomez-Maldonado present address: Forest Products Development Center in the School of Forestry and Wildlife Sciences at Auburn University.

Haley Stephens present address: Department of Biological Sciences, College of Science and Mathematics at Auburn University.

Haley Stephens and Diego Gomez-Maldonado contributed equally to this work.

School of Forestry and Wildlife Sciences, Auburn University, Auburn, AL, United States

Haley Stephens, Diego Gomez-Maldonado, Andrea Camarano Eula, Iris Beatriz Vega Erramuspe,

Soledad Peresin, and Sarah Zohdy

Forest Products Development Center, School of Forestry and Wildlife Sciences, Auburn University, Auburn, AL, United States

Diego Gomez-Maldonado, Iris Beatriz Vega Erramuspe, Soledad Peresin

Centers for Disease Control and Prevention, Atlanta, GA, United States

Alice Sutcliffe, Ellen Dotson 
2. Fontenille D, Meunier JY, Nkondjio CA, Tchuinkam T. Use of circumsporozoite protein enzyme-linked immunosorbent assay compared with microscopic examination of salivary glands for calculation of malaria infectivity rates in mosquitoes (Diptera : culicidae) from Cameroon. Journal of Medical Entomology. 2001;38(3):451-4.

3. Lequin RM. Enzyme Immunoassay (EIA)/Enzyme-Linked Immunosorbent Assay (ELISA). Clinical Chemistry. 2005;51(12):2415-8.

4. Coppi A, Natarajan R, Pradel G, Bennett BL, James ER, Roggero MA. The malaria circumsporozoite protein has two functional domains, each with distinct roles as sporozoites journey from mosquito to mammalian host. J Exp Med.2011. p. 341-56.

5. Gan SD, Patel KR. Enzyme Immunoassay and Enzyme-Linked Immunosorbent Assay. Journal of Investigative Dermatology. 2013;133(9):E10-E2.

6. Durnez L, Van Bortel W, Denis L, Roelants P, Veracx A, Trung HD, et al. False positive circumsporozoite protein ELISA: a challenge for the estimation of the entomological inoculation rate of malaria and for vector incrimination. Malar J. 2011;10:195.

7. Sia SK, Linder V, Parviz BA, Siegel A, Whitesides GM. An integrated approach to a portable and low-cost immunoassay for resource-poor settings. Angewandte Chemie-International Edition. 2004;43(4):498-502.

434 Angewandte Chemie-International Edition. 2010;49(28):4771-4. 
435 9. Maxwell EJ, Mazzeo AD, Whitesides GM. Paper-based electroanalytical devices for accessible diagnostic 436 testing. Mrs Bulletin. 2013;38(4):309-14.

437 10. Evans E, Moreira Gabriel EF, Benavidez TE, Tomazelli Coltro WK, Garcia CD. Modification of microfluidic 438 paper-based devices with silica nanoparticles. Analyst. 2014;139(21):5560-7.

439 11. Carrilho E, Martinez AW, Whitesides GM. Understanding Wax Printing: A Simple Micropatterning Process 440 for Paper-Based Microfluidics. Analytical Chemistry. 2009;81(16):7091-5.

441 12. Pandey JK, Takagi H, Nakagaito AN, Kim H-J. Handbook of polymer nanocomposites : processing, 442 performance and application. Volume C, Polymer nanocomposites of cellulose nanoparticles.

443 13. Smook G. Handbook For Pulp and Paper Technologists (The SMOOK Book), Fourth Edition: TAPPI 444 PRESS; 2016.

445 14. Moon RJ, Martini A, Nairn J, Simonsen J, Youngblood J. Cellulose nanomaterials review: structure, 446 properties and nanocomposites. Chemical Society Reviews. 2011;40(7):3941-94.

447 15. Kumar M, Muzzarelli RAA, Muzzarelli C, Sashiwa H, Domb AJ. Chitosan chemistry and pharmaceutical 448 perspectives. Chemical Reviews. 2004;104(12):6017-84.

449 16. Younes E, Haas GP, Dezso B, Ali E, Maughan RL, Kukuruga MA, et al. LOCAL TUMOR-IRRADIATION 450 AUGMENTS THE RESPONSE TO IL-2 THERAPY IN A MURINE RENAL ADENOCARCINOMA. Cellular 451 Immunology. 1995;165(2):243-51.

452 17. Younes I, Rinaudo M. Chitin and chitosan preparation from marine sources. Structure, properties and 453 applications. Marine DrugsMarch 2015. p. 1133-74. 
454 18. Kumar M. A review of chitin and chitosan applications. Reactive \& Functional Polymers. 2000;46(1):1-27.

455 19. Gabriel EFM, Garcia PT, Cardoso TMG, Lopes FM, Martins FT, Coltro WKT. Highly sensitive colorimetric 456 detection of glucose and uric acid in biological fluids using chitosan-modified paper microfluidic devices. Analyst. $457 \quad 2016 ; 141(15): 4749-56$.

458 20. Wirtz R. Plasmodium vivax Sporozoite ELISA Reagent Kit. In: Control CFD, editor.: American Type 459 Culture Collection (ATCC); 2017. p. 1-12.

460 21. Sutcliffe AC, Irish SR, Rogier E, Finney M, Zohdy S, Dotson EM. Adaptation of ELISA detection of 461 Plasmodium falciparum and Plasmodium vivax circumsporozoite proteins in mosquitoes to a multiplex bead-based 462 immunoassay.

463 22. Young JF, Hockmeyer WT, Gross M, Ballou WR, Wirtz RA, Trosper JH, et al. Expression of Plasmodium 464 falciparum circumsporozoite proteins in Escherichia coli for potential use in a human malaria vaccine. Science. $465 \quad 1985 ; 228(4702): 958-62$.

466 23. Sim KL, Hoffman S, Arevalo M, Herrera S, inventorsUnique DNA and polypeptide sequences based on the 467 circumsporozoite protein of Plasmodium vivax. US2006.

468 24. Orelma H, Filpponen I, Johansson L-S, Laine J, Rojas OJ.

469 25. Beier JC, Onyango FK, Koros JK, Ramadhan M, Ogwang R, Wirtz RA, et al. Quantitation of malaria 470 sporozoites transmitted in vitro during salivation by wild Afrotropical Anopheles. Medical and Veterinary $471 \quad$ Entomology. 1991;5(1):71-9.

472 26. Israelachvili JN. Intermolecular and surface forces. 3rd ed. Burlington, MA: Academic Press; 2011. xxx, 674 $473 \quad$ p. p. 
474 27. Salas C, Nypelö T, Rodriguez-Abreu C, Carrillo C, Rojas OJ. Nanocellulose properties and applications in 475 colloids and interfaces. Current Opinion in Colloid \& Interface Science. 2014;19(5):383-96.

476 28. Mishima T, Hisamatsu M, York WS, Teranishi K, Yamada T. Adhesion of $\beta$-d-glucans to cellulose. 477 Carbohydrate Research. 1998;308(3):389-95.

478

479 
Page $\mathbf{2 3}$ of $\mathbf{2 3}$ 


\section{Figures}
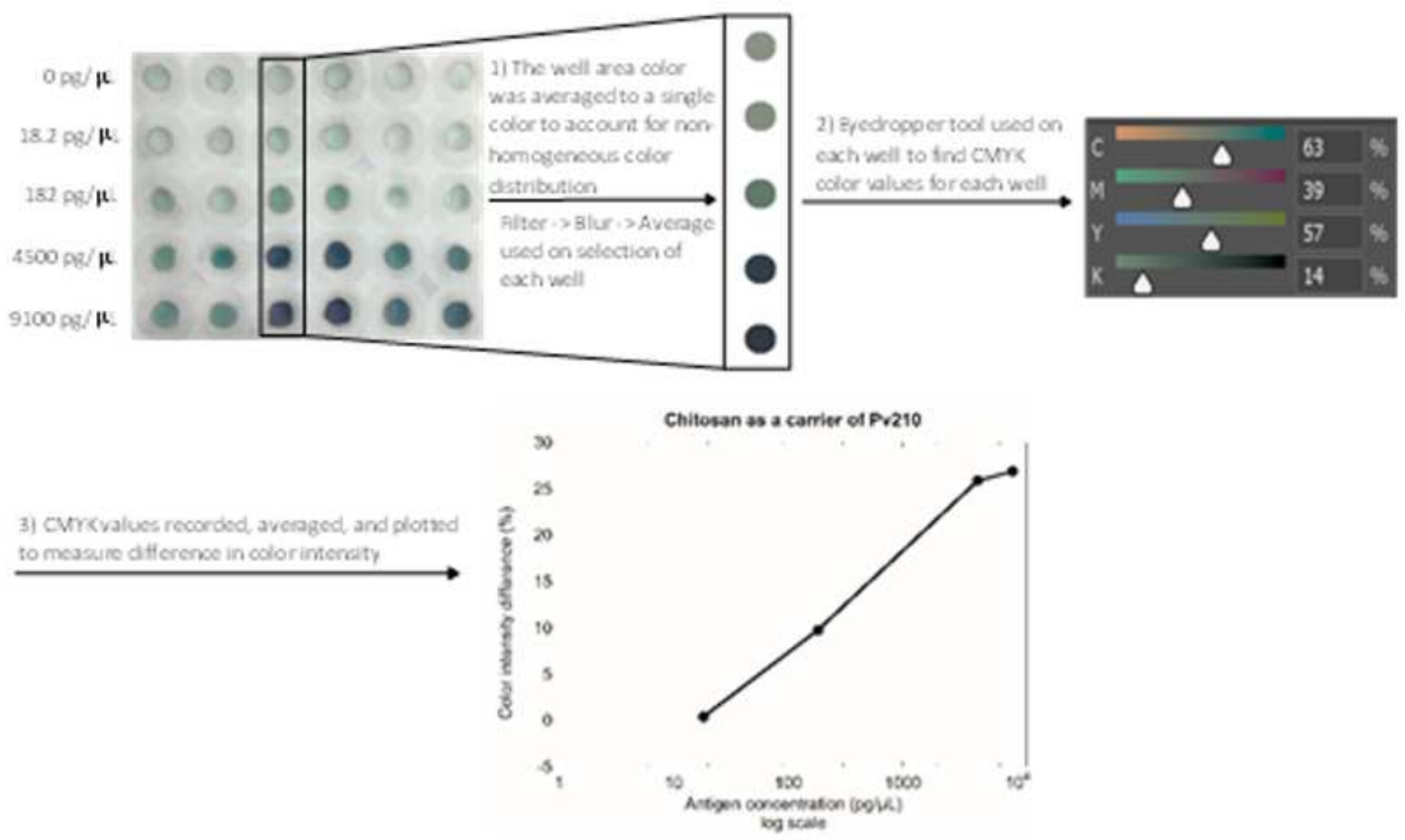

\section{Figure 1}

Workflow showing conversion of cellphone photograph to numerical color data. CMYK changes in each well were averaged to one single value, which was then identified as the color intensity difference percentage. This color intensity value was then plotted against antigen concentration to determine the relationship between the two. 


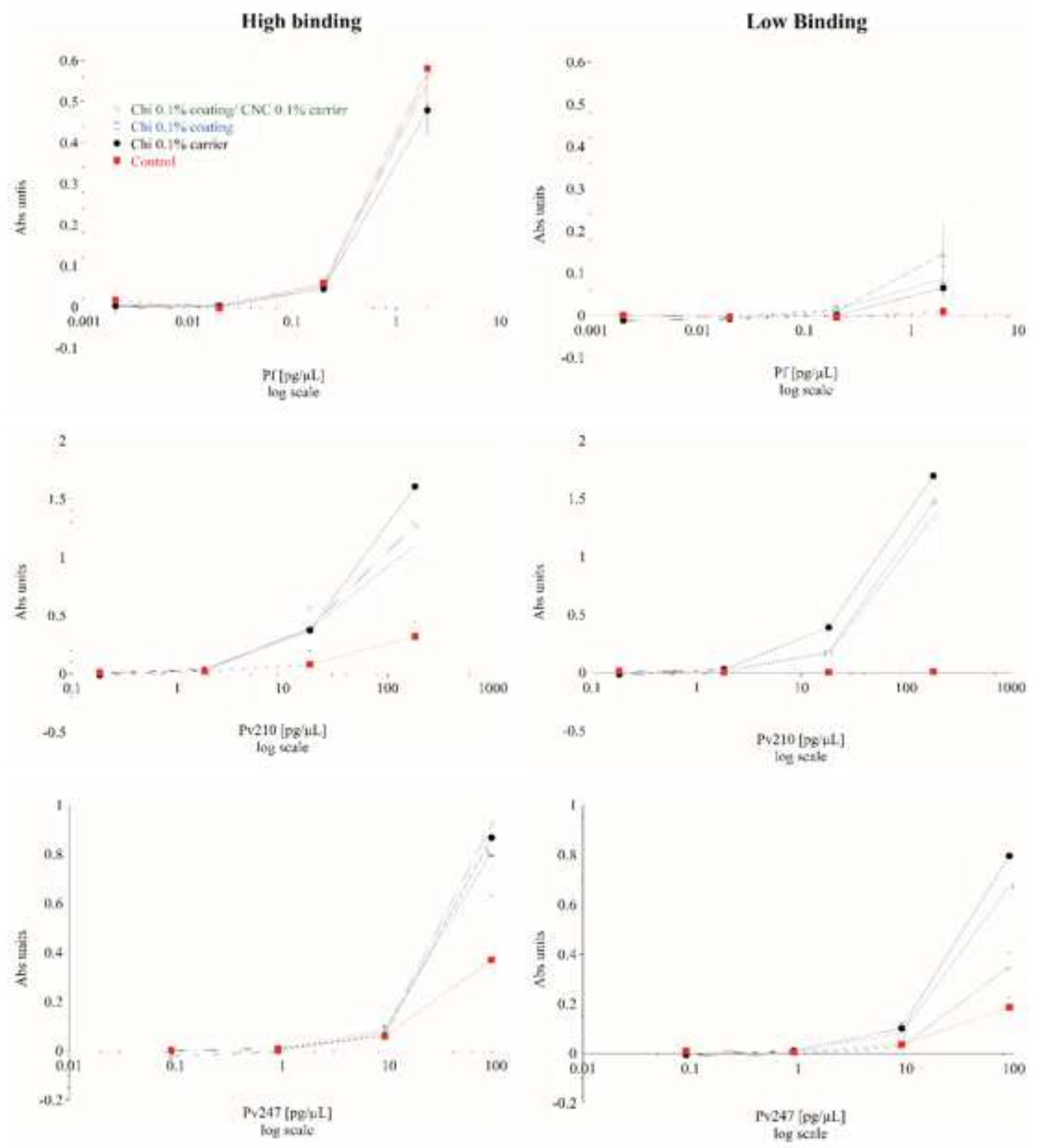

Figure 2

ELISA results for Pf, Pv210 and Pv247 run on high-binding (MaxiSorp ${ }^{\mathrm{TM}}$ ) and ultra-low binding(Corning ${ }^{\circledR}$ 3474) plates. The conditions tested were chitosan as antibody carrier or well-coating (Chi $0.1 \%$ coating), chitosan as an antibody carrier (Chi $0.1 \%$ carrier) and chitosan as well-coating and cellulose nanocrystal as a carrier (Chi $0.1 \%$ coating / CNC $0.1 \%$ carrier). The samples and controls were run at four concentrations: the standard cs-ELISA recombinant protein working solution concentrations and three 10fold dilutions. The working solution concentrations were: $2 \mu \mathrm{g} / \mu \mathrm{l}$ for Pf, $182 \mu \mathrm{g} / \mu \mathrm{l}$ for Pv210 and $91 \mu \mathrm{g} / \mu \mathrm{l}$ for Pv247. The absorbance was measured at $405 \mathrm{~nm}$. Samples and controls were run in triplicate and each assay was repeated three times. Bars represent $S D, n=9$. 


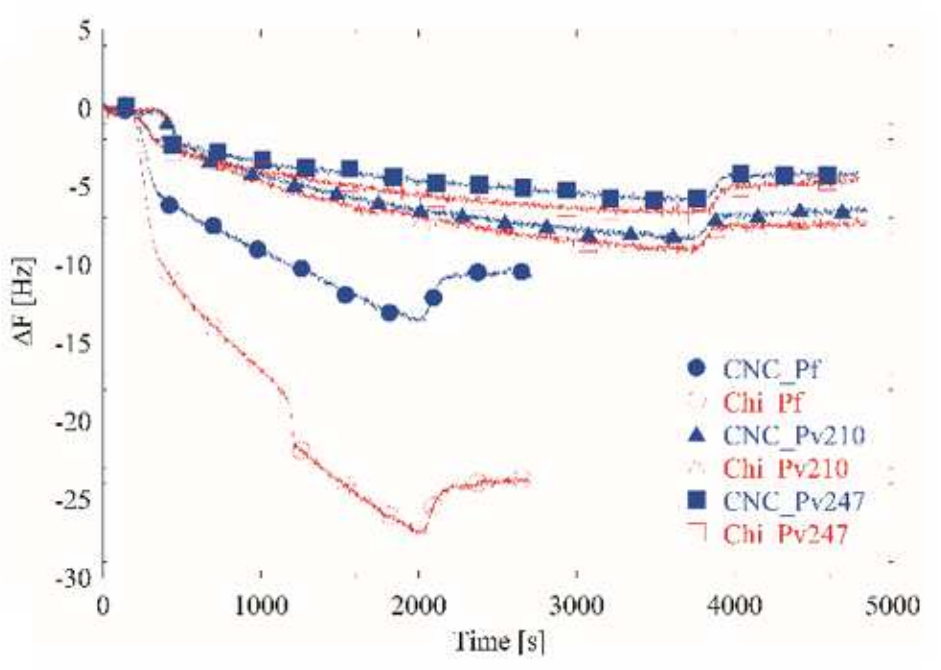

(A)

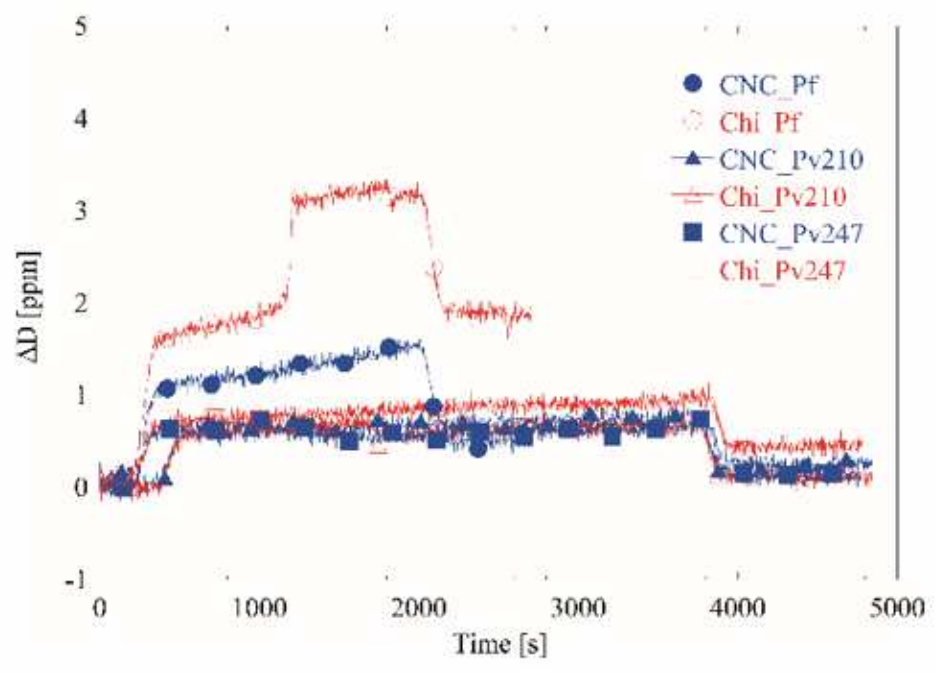

(B)

Figure 3

QCM-D results of adsorption of Pf, Pv210, and Pv247 antibodies in model film surfaces of cellulose nanocrystals (CNC) and chitosan (Chi). The surfaces were analyzed over precoated gold sensors with PEI with $0.1 \% \mathrm{CNC}$ or chitosan solutions at $3000 \mathrm{rpm}$. Flow concentrations of the antibodies were $2 \mu \mathrm{g} / \mu \mathrm{l}$ for $\mathrm{Pf}, 182 \mu \mathrm{g} / \mu \mathrm{l}$ for Pv210, and $91 \mu \mathrm{g} / \mu \mathrm{l}$ for Pv247 in PBS and flow of $50 \mu \mathrm{L} / \mathrm{min}$. 


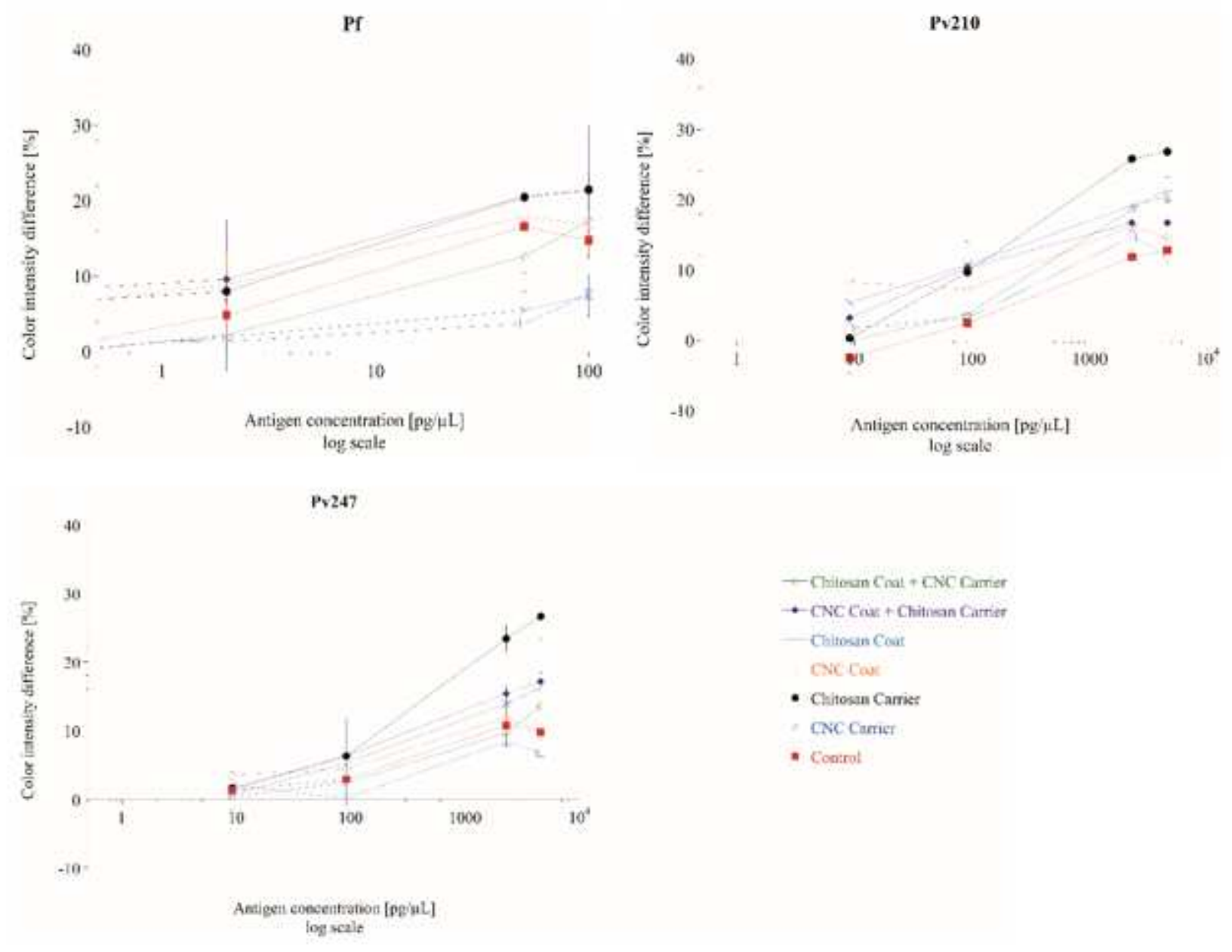

\section{Figure 4}

Paper csELISA results for all antibody carrier and well-coating assays. Recombinant proteins were tested at concentrations as follows: Pf at $0 \mathrm{pg} / \mu \mathrm{L}, 0.2 \mathrm{pg} / \mu \mathrm{L}, 2 \mathrm{pg} / \mu \mathrm{L}, 50, \mathrm{pg} / \mu \mathrm{L}$, and $100 \mathrm{pg} / \mu \mathrm{L}, \mathrm{Pv} 210$ at $0 \mathrm{pg} /$ $\mu \mathrm{L}, 18.2 \mathrm{pg} / \mu \mathrm{L}, 182 \mathrm{pg} / \mu \mathrm{L}, 4500 \mathrm{pg} / \mu \mathrm{L}$, and $9100 \mathrm{pg} / \mu \mathrm{L}$, and Pv247 at $0 \mathrm{pg} / \mu \mathrm{L}, 9.1 \mathrm{pg} / \mu \mathrm{L}, 91 \mathrm{pg} / \mu \mathrm{L}$, $2275 \mathrm{pg} / \mu \mathrm{L}$, and $4550 \mathrm{pg} / \mu \mathrm{L}$. Data were plotted and converted to log-scale as a graph of color intensity difference versus concentration of the recombinant protein. The change in color intensity for each concentration was calculated as the difference between the average CMYK value and the corresponding blank (in percentage). 


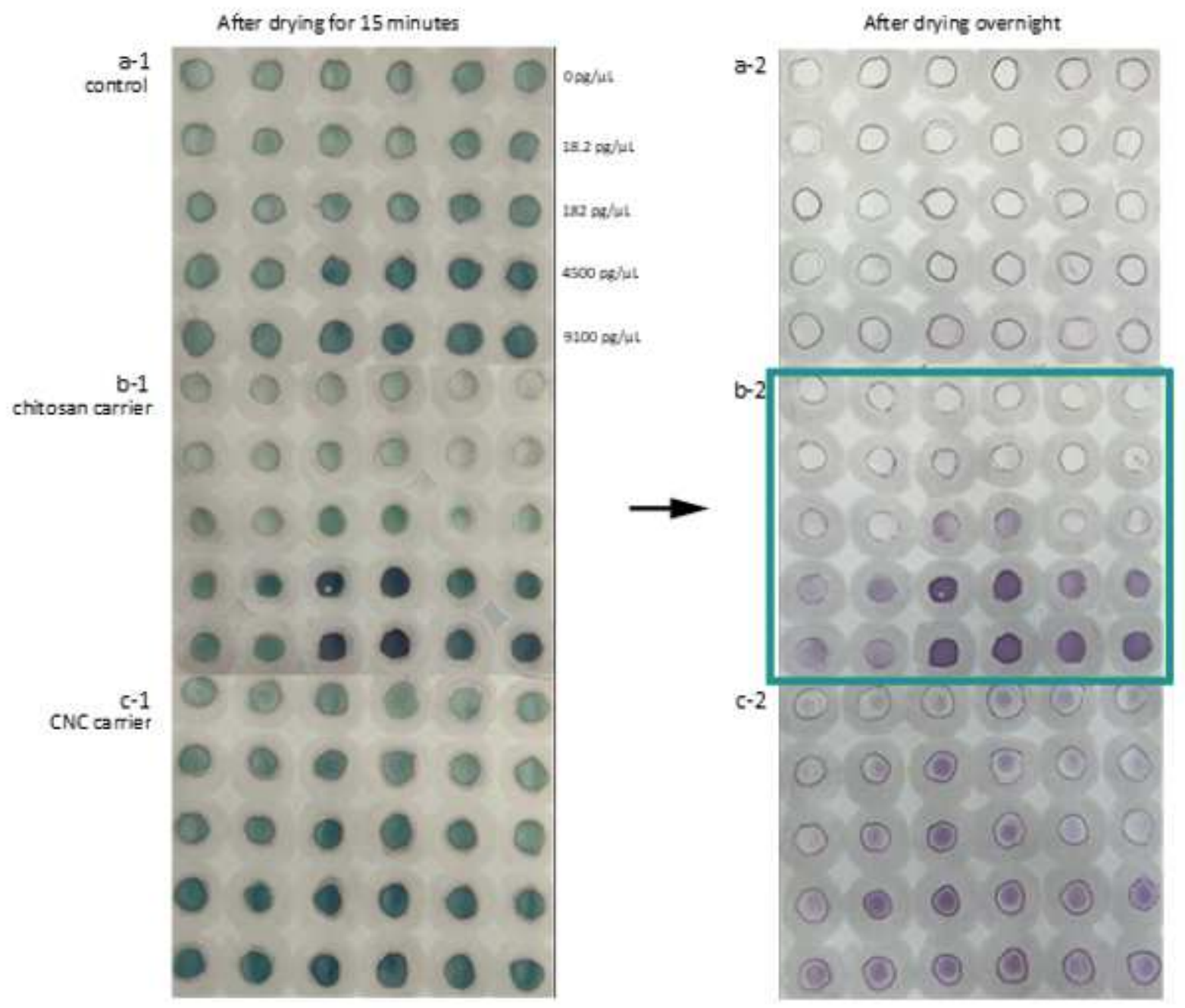

\section{Figure 5}

Photographs of paper-plate assays were taken using a cellphone camera after drying for 15 minutes, and overnight. Significant differences can be seen in the distribution of color and overall well homogeneity between the three different assays. Both bio-based material assays showed a notable improvement over the control, with the chitosan carrier assay showing the clearest visible difference in increasing antigen concentration.

\section{Supplementary Files}

This is a list of supplementary files associated with this preprint. Click to download.

- csELISAmanuscriptsupportinginformation.docx 